\title{
Predictive value of creatine kinase MB for contrast-induced acute kidney injury among myocardial infarction patients
}

Wen Wei ${ }^{1,2,3+}$, Lingyu Zhang ${ }^{4 \dagger}$, Yunhan Zhang ${ }^{5+}$, Ronghui Tang ${ }^{6+}$, Miao Zhao ${ }^{6+}$, Zhidong Huang ${ }^{1}$, Jin Liu', Danyuan Xu' ${ }^{1}$, Yibo He ${ }^{1}$, Bo Wang ${ }^{1}$, Haozhang Huang ${ }^{3}$, Qiang Li', Mengfei Lin ${ }^{4}$, Yong Liu ${ }^{1,3,7}$, Kaihong Chen ${ }^{8^{*}}$ and Shiqun Chen ${ }^{1 *}$

\begin{abstract}
Background: Predictive value of creatine kinase MB (CK-MB) for contrast-induced acute kidney injury (CI-AKI) among myocardial infarction (MI) patients has rarely been reported. We aim to evaluate the predictive value of CK-MB for $\mathrm{Cl}$ AKl among Ml patients.

Methods: Totally, $1131 \mathrm{Ml}$ patients were included from the REduction of rlsk for Contrast-Induced Nephropathy (REICIN) study. The peak CK-MB before coronary angiography (CAG) was chosen. The study population was divided into two groups by log-transformed CK-MB cut-off point. The association between CK-MB and CI-AKI was tested by multivariable logistic regression. CK-MB was integrated with Age, creatinine and ejection fraction (ACEF) score and Mehran risk score (MRS) to evaluate the additive value of CK-MB. The integrated models were validated internally by the bootstrap method and externally by the PREdictive Value of COntrast voluMe to creatinine Clearance Ratio (PRECOMIN) study data set.
\end{abstract}

Results: Overall, 62(5.48\%) patients developed CI-AKI, patients with CK-MB point $>4.7$ displayed a higher incidence of $\mathrm{Cl}$-AKI than those without $(11.9 \%$ vs. $4.0 \%, p<0.001)$. CK-MB point $>4.7$ was independently associated with $\mathrm{Cl}-\mathrm{AKI}$ (adjusted OR: $3.40,95 \%$ Cl: 1.93-5.98, $p<0.001)$. The additions of CK-MB to ACEF score, Mehran score A and Mehran score $B$ resulted in increases in C-statistics, which ranged from 0.680 to $0.733(p=0.046), 0.694$ to $0.727(p=0.091)$, 0.704 to $0.734(p=0.102)$, respectively. Internal validation also showed increases in C-statistics, and external validation performed well in discrimination and calibration.

Conclusions: Preprocedural peak CK-MB was a predictor of Cl-AKI among MI patients.

Keywords: Creatine kinase-MB, Contrast-induced acute kidney injury, Predictive, Myocardial infarction

*Correspondence: chenkaihong1964@163.com; shiqunchen@126.com †Wen Wei, Lingyu Zhang, Yunhan Zhang, Ronghui Tang and Miao Zhao have contributed equally to this work

${ }^{1}$ Department of Cardiology, Guangdong Provincial Key Laboratory of Coronary Heart Disease Prevention, Guangdong Cardiovascular Institute, Guangdong Provincial People's Hospital, Guangdong Academy of Medical Sciences, Guangzhou 510080, China

${ }^{8}$ Department of Cardiology, Longyan First Affiliated Hospital of Fujian Medical University, Longyan 364000, China

Full list of author information is available at the end of the article

\section{Introduction}

Contrast-induced acute kidney injury (CI-AKI) is the third most common cause of hospital-acquired renal failure, with an incidence of $11 \%$ [1], and is associated with poor short- and long-term outcomes [2-5]. As an important and readily available cardiac biomarker, creatine kinase $\mathrm{MB}(\mathrm{CK}-\mathrm{MB})$ has long been used in the diagnosis of acute myocardial infarction (AMI) because of its good cost-performance ratio and simplicity [6]. CK-MB has 
also been indicated to improve clinical risk prediction of postoperative acute kidney injury (AKI) among patients undergoing cardiac surgery [7]. In a study of 257 patients, Katarzyna ZR et al. found that increased CK-MB and Red Cell Distribution Width (RDW) are associated with higher risk of CI-AKI among patients with AMI [8]. However, few studies have investigated the independent predictive value of CK-MB in CI-AKI among myocardial infarction (MI) patients.

Therefore, we aim to evaluate the independent predictive utility of CK-MB to CI-AKI risk and determine whether CK-MB can add predictive information to the traditional risk models for determining CI-AKI among MI patients undergoing coronary angiography (CAG) or percutaneous coronary intervention (PCI).

\section{Methods}

\section{Data sources and study population}

This study included $1131 \mathrm{MI}$ patients from the multicenter prospective REduction of rIsk for ContrastInduced Nephropathy (REICIN) study from January 2013 to June 2016 (trial registration: ClinicalTrials.gov NCT01402232). Only adult patients ( $\geq 18$ years of age; referred to CAG or PCI) with providing written informed consent were studied. This study was conducted in accordance with the Declaration of Helsinki and was approved by the Research Ethics Committee of Guangdong Provincial People's Hospital, Guangdong Academy of Medical Sciences (No. GDREC2012141H).Follow-up data was monitored and recorded by trained nurses and research assistants through outpatient interviews and telephones.

\section{Variables and study endpoint}

Biochemistry data CK-MB was evaluated on admission, at 3-h intervals in the first $24 \mathrm{~h}$, and daily in the first 3 days following admission. The peak CK-MB before CAG or PCI was chosen. Immunosuppressive method was used to determine the activity of CK-MB and was applied to each sub-center. Due to its non-normal distribution, the CK-MB variable was log-transformed. Receiver operating characteristic (ROC) curve was used to determine the cut-off point of optimal prognostic performance. Then the CK-MB was for the next analysis as a categorical variable based on the cut-off point.

Serum creatinine $(\mathrm{Scr})$ concentration was measured at admission and within 24, 48 and $72 \mathrm{~h}$ after CAG or PCI. Other biochemical indicators were evaluated on admission. The echocardiography examination was used to evaluate the left ventricular ejection fraction (LVEF).

The primary endpoint was $\mathrm{CI}-\mathrm{AKI}_{0350}$, defined as an increase in the Scr by over $0.3 \mathrm{mg} / \mathrm{dL}$ or over $50 \%$ from baseline within the first $48 \mathrm{~h}$ after the CAG [9]. The secondary endpoint was 3-year all-cause mortality.

\section{ACEF score and Mehran risk score (MRS)}

Age, creatinine and ejection fraction (ACEF) score was calculated by evaluating age, Scr and LVEF [10]. MRS was calculated by evaluating the presence of hypotension, congestive heart failure(CHF), anemia, and diabetes mellitus(DM), the use of intra-aortic balloon pump(IABP), age $>75$ years, the amount of contrast medium, and the basal renal function. There are two types of MRS: Mehran score A using Scr as a criterion for renal function, and Mehran score B using estimated glomerular filtration rate (eGFR) [11].

\section{Validation cohort}

The PREdictive Value of COntrast voluMe to creatinine Clearance Ratio (PRECOMIN, trial registration:ClinicalTrials.gov NCT01400295) study [12] was a prospective single-center observational study that reviewed all consecutive patients $(\mathrm{n}=3369)$ undergoing CAG and/or PCI between January 2010 and October 2012 according to the institutional protocol. The PRECOMIN study included 1312 MI patients, of which 511 samples had no data deletion. Among 511 patients, $58(11.35 \%)$ patients fulfilled the diagnostic criteria for CI-AKI ${ }_{0350}$.

\section{Statistical analyses}

Continuous variables were expressed as mean (standard deviation $[\mathrm{SD}]$ ) or medians interquartile range (IQRs), and discrete variables were expressed as frequency counts and percentages. The differences in variables among groups were evaluated by the t-test or chi-square test. The association between CK-MB and CI-AKI was tested by univariable and multivariable logistic regression. And then, CK-MB was integrated with ACEF score and MRS to compare the predictive power of before and after addition. The performances were evaluated based on discrimination and calibration. Discrimination was evaluated with the ROC curve and expressed by the $\mathrm{C}$-statistic. The C-statistics were compared by the Delong test. We also compared the models using the continuous net reclassification index (NRI) and integrated discrimination and improvement (IDI). The calibration of these models was described by the Hosmer-Lemeshow test.

To evaluate the stability of the integrated models, these models were validated internally using 1000 bootstrap samples and externally validated in the PRECOMIN study data set. We calculated an optimal bootstrap-corrected C-statistic as described by Riley et al. by fitting the prediction model in each of the 1000 bootstrap samples 
Table 1 Comparison of clinical characteristics between patients with and without elevated CK-MB

\begin{tabular}{|c|c|c|c|c|}
\hline Characteristic & Overall $(n=1131)$ & $\log (C K-M B) \leq 4.70(n=920)$ & $\begin{array}{l}\log (C K-M B)>4.70 \\
(n=211)\end{array}$ & $p$-value \\
\hline \multicolumn{5}{|c|}{ Demographic characteristics } \\
\hline Age (years) & $60.87 \pm 12.05$ & $60.90 \pm 12.00$ & $60.71 \pm 12.28$ & 0.834 \\
\hline Male gender & $932(82.40 \%)$ & $761(82.72 \%)$ & $171(81.04 \%)$ & 0.634 \\
\hline Weight (kg) & $65.74 \pm 10.84$ & $65.71 \pm 10.96$ & $65.89 \pm 10.31$ & 0.842 \\
\hline $\mathrm{BMI}\left(\mathrm{kg} / \mathrm{m}^{2}\right)$ & $23.91 \pm 3.21$ & $23.91 \pm 3.24$ & $23.93 \pm 3.06$ & 0.958 \\
\hline \multicolumn{5}{|c|}{ Medical history and clinical condition } \\
\hline $\mathrm{Cl}-\mathrm{AKI}$ & $62(5.48 \%)$ & $37(4.02 \%)$ & $25(11.85 \%)$ & $<0.001$ \\
\hline Smoking history & $542(47.92 \%)$ & $424(46.09 \%)$ & $118(55.92 \%)$ & 0.012 \\
\hline DM & $295(26.08 \%)$ & $243(26.41 \%)$ & $52(24.64 \%)$ & 0.659 \\
\hline Hypertension & $562(49.69 \%)$ & $471(51.20 \%)$ & $91(43.13 \%)$ & 0.042 \\
\hline Hyperlipidemia & $139(12.29 \%)$ & $106(11.52 \%)$ & $33(15.64 \%)$ & 0.127 \\
\hline CKD & $607(53.67 \%)$ & $496(53.91 \%)$ & $111(52.61 \%)$ & 0.790 \\
\hline $\mathrm{CHF}$ & $460(40.67 \%)$ & $368(40.00 \%)$ & $92(43.60 \%)$ & 0.377 \\
\hline Anterior infarction & $399(35.28 \%)$ & $291(31.63 \%)$ & $108(51.18 \%)$ & $<0.001$ \\
\hline Hypotension & $86(7.60 \%)$ & $59(6.41 \%)$ & $27(12.80 \%)$ & 0.003 \\
\hline Anemia & $321(28.38 \%)$ & $275(29.89 \%)$ & $46(21.80 \%)$ & 0.023 \\
\hline IABP & $42(3.71 \%)$ & $28(3.04 \%)$ & $14(6.64 \%)$ & 0.022 \\
\hline LVEF (\%) & $55.17 \pm 11.34$ & $55.91 \pm 11.37$ & $52.02 \pm 10.69$ & $<0.001$ \\
\hline \multicolumn{5}{|l|}{ Procedure } \\
\hline Emergent $\mathrm{PCl}$ & $976(86.30 \%)$ & $782(85.00 \%)$ & 194 (91.94\%) & 0.011 \\
\hline \multicolumn{5}{|l|}{ Laboratory examination } \\
\hline $\mathrm{GLU}(\mathrm{mmol} / \mathrm{L})$ & $8.16 \pm 3.89$ & $8.03 \pm 3.88$ & $8.71 \pm 3.91$ & 0.022 \\
\hline $\mathrm{Hb}(\mathrm{g} / \mathrm{L})$ & $128.05 \pm 20.46$ & $128.73 \pm 20.21$ & $125.05 \pm 21.34$ & 0.020 \\
\hline hct & $40.62 \pm 5.87$ & $40.39 \pm 5.89$ & $41.64 \pm 5.68$ & 0.006 \\
\hline $\operatorname{Scr}(\mathrm{mg} / \mathrm{dl})$ & $95.37 \pm 40.66$ & $96.20 \pm 42.43$ & $91.72 \pm 31.62$ & 0.148 \\
\hline $\mathrm{CCR}(\mathrm{ng} / \mathrm{ml})$ & $73.70 \pm 28.88$ & $73.36 \pm 28.86$ & $75.18 \pm 28.98$ & 0.410 \\
\hline $\mathrm{eGFR}\left(\mathrm{ml} / \mathrm{min} / 1.73 \mathrm{~mm}^{2}\right)$ & $80.98 \pm 26.73$ & $80.69 \pm 26.89$ & $82.26 \pm 26.04$ & 0.441 \\
\hline $\mathrm{BUN}(\mathrm{mmol} / \mathrm{L})$ & $5.00[3.90,6.50]$ & $4.91[3.90,6.48]$ & $5.20[4.03,6.58]$ & 0.143 \\
\hline $\mathrm{HDL}-\mathrm{C}(\mathrm{mmol} / \mathrm{L})$ & $1.00 \pm 0.28$ & $0.98 \pm 0.27$ & $1.08 \pm 0.33$ & $<0.001$ \\
\hline $\mathrm{ALB}(\mathrm{g} / \mathrm{L})$ & $36.10 \pm 5.15$ & $36.07 \pm 5.12$ & $36.23 \pm 5.31$ & 0.712 \\
\hline \multicolumn{5}{|c|}{ Treatment during hospitalization } \\
\hline ACEI/ARB & $145(12.82 \%)$ & $133(14.46 \%)$ & $12(5.69 \%)$ & 0.001 \\
\hline Beta-blockers & $611(54.02 \%)$ & $532(57.83 \%)$ & 79 (37.44\%) & $<0.001$ \\
\hline $\mathrm{CCB}$ & $89(7.87 \%)$ & $84(9.13 \%)$ & $5(2.37 \%)$ & 0.002 \\
\hline Statins & $912(80.64 \%)$ & $764(83.04 \%)$ & $148(70.14 \%)$ & $<0.001$ \\
\hline Hypoglycemic drugs & $125(11.05 \%)$ & $115(12.50 \%)$ & $10(4.74 \%)$ & 0.002 \\
\hline Diuretic & $261(23.08 \%)$ & $201(21.85 \%)$ & $60(28.44 \%)$ & 0.050 \\
\hline Contrast dose(ml) & $113.80 \pm 50.15$ & $112.14 \pm 50.05$ & $121.02 \pm 50.08$ & 0.021 \\
\hline
\end{tabular}

$C K-M B$ creatine kinase isoenzymes/creatine kinase-MB, BMI body mass index, $C l-A K I$ contrast-induced acute kidney injury, DM diabetes mellitus, $C K D$ chronic kidney disease, CHF congestive heart failure, IABP intra-aortic ballon pump, LVEF left ventricular ejection fraction, $P C I$ percutaneous coronary intervention, $G L U$ blood glucose, $\mathrm{Hb}$ hemoglobin, $h c t$ hematocrit, Scr serum creatinine, CCR Creatinine Clearance Rate, eGFR estimated glomerular filtrationrate, $B U N$ blood urea nitrogen, $H D L-C$ high density lipoprotein cholesterol, $A L B$ albumin, $A C E I / A R B$ angiotensin-converting enzyme inhibitor/angiotensin receptor blocker, $C C B$ calcium channel blocker

[13]. External validation was furthermore assessed by both discrimination and calibration.

All analyses were performed with $\mathrm{R}$ software (version 4.0.3; R Foundation for Statistical Computing, Vienna, Austria). A two-sided $p$-value $<0.05$ indicated significance for all analyses.

\section{Result}

Baseline clinical characteristics and outcomes

From January 2013 to June 2016, a total of 1131 consecutive MI patients who underwent CAG or PCI were included. The mean age was $60.87 \pm 12.05$ years, and 932 patients $(82.40 \%)$ were males. $62(5.48 \%)$ patients fulfilled 
Table 2 Univariate and multivariate logistic regression analysis of $\mathrm{Cl}-\mathrm{AKI}_{0305}$

\begin{tabular}{llrlllr}
\hline & \multicolumn{2}{l}{ Univariate } & & & Multivariate & \\
\cline { 2 - 3 } \cline { 6 - 7 } & OR $(\mathbf{9 5 \%} \% \mathbf{C l})$ & $\boldsymbol{p}$-value & & OR(95\%Cl) & $\boldsymbol{p}$-value \\
\hline Age(years) & $1.05(1.02,1.07)$ & $<0.001$ & & $1.04(1.02,1.07)$ & 0.001 \\
Scr & $1.01(1.01,1.01)$ & $<0.001$ & & $1.01(1.00,1.01)$ & $<0.001$ \\
Contrast dose $(\mathrm{ml})$ & $1.00(1.00,1.01)$ & 0.419 & & $1.00(1.00,1.01)$ & 0.384 \\
DM & $1.37(0.79,2.38)$ & 0.256 & & $1.28(0.72,2.28)$ & 0.404 \\
LVEF (\%) & $0.96(0.94,0.98)$ & $<0.001$ & & $0.98(0.96,1.00)$ & 0.036 \\
CK-MB & $3.21(1.89,5.46)$ & $<0.001$ & & $3.40(1.93,5.98)$ & $<0.001$ \\
\hline
\end{tabular}

Cl-AKI contrast-induced acute kidney injury, Scr serum creatinine, DM diabetes mellitus, $L V E F$ left ventricular ejection fraction, $C K-M B$ creatine kinase isoenzymes/creatine kinase-MB

the diagnostic criteria for CI-AKI $\mathrm{A350}_{0}$. The cut-off point of $\log$-transformed CK-MB for the best predictive value of CI-AKI was 4.7. Therefore, patients were divided into two groups based on the cut-off point: 920 (81.34\%) patients with log-transformed CK-MB $\leq 4.7$, and 211(18.66\%) patients with log-transformed CK-MB $>4.7$. All of the baseline clinical characteristics of the patients are shown in Table 1. Overall, there were 607(53.67\%) patients with chronic kidney disease (CKD), 295(26.08\%) patients with DM and 562 (49.69\%) patients with hypertension.

Compared with patients with log-transformed $\mathrm{CK}-\mathrm{MB} \leq 4.7$, patients with $\log$-transformed CK-MB $>4.7$ demonstrated lower LVEF (52 \pm 11 vs. $56 \pm 11, p<0.001$ ) and higher random plasma glucose $(8.7 \pm 3.9$ vs. $8.0 \pm 3.9 \mathrm{mmol} / \mathrm{l}, p=0.022)$. These patients also showed higher incidences of hypotension on the day of admission $(12.8 \%$ vs. $6.4 \%, p=0.003)$, anterior infarction $(51.2 \%$ vs. $31.6 \%, p<0.001)$ and IABP $(6.6 \%$ vs. $3.0 \%, p=0.022)$. Nonetheless, there were no significant differences in the incidences of CKD, DM and CHF between the two groups $(p>0.05)$ (Table 1$)$.

\section{Predictive value of CK-MB in $\mathrm{Cl}-\mathrm{AKI}$}

Compared with patients with log-transformed $\mathrm{CK}-\mathrm{MB} \leq 4.7$, patients with log-transformed CK-MB $>4.7$ displayed a significantly greater incidence of CI-AKI ( $11.9 \%$ vs. $4.0 \%, p<0.001$, Table 1$)$. The area under the CK-MB curve in relation to CI-AKI was 0.625 (95\% confidence interval [CI]: 0.550 to 0.701 ), and a Hosmer-Lemeshow $\chi^{2}$ statistic of $11.37(p=0.182)$. Univariate logistic regression analysis indicated that logtransformed CK-MB $>4.7$ was significantly correlated
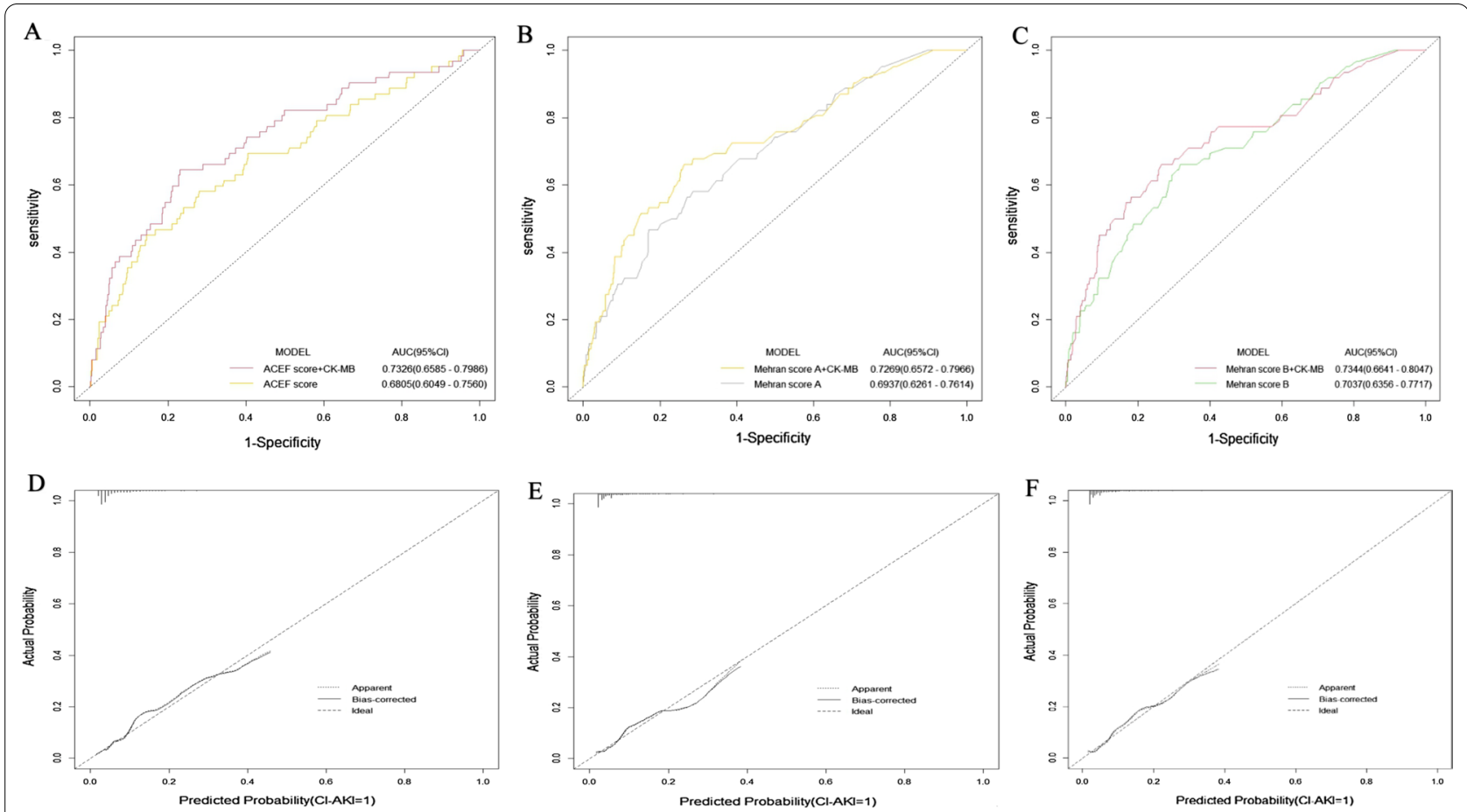

Fig. 1 The performance of CK-MB before and after adding ACEF score and MRS in REICIN study data set. a The ROC curves of ACEF sCore and ACEF score + CK-MB for CI-AKI. $\mathbf{b}$ The ROC curves of Mehran score A and Mehran score A+CK-MB for CI-AKI. c The ROC curves of Mehran score B and Mehran score B + CK-MB for CI-AKI. d The bootstrap curve of ACEF score + CK-MB integrated model for CI-AKI. e The bootstrap curve of Mehran score A+CK-MB integrated model for Cl-AKI. f The bootstrap curve of Mehran score B + CK-MB integrated model for Cl-AKI. AUC area under the receiver operating characteristic curve, $R O C$ receiver operator characteristic, CK-MB creatine kinase isoenzymes/creatine kinase-MB, MRS Mehran risk score, ACEF Age, creatinine and ejection fraction, REICIN REduction of rlsk for Contrast-Induced Nephropathy 
Table 3 Discrimination and calibration analysis of CK-MB added to models in REICIN study data set

\begin{tabular}{|c|c|c|c|c|c|c|c|c|c|}
\hline & C-statistics & $p$-value & NRI & $p$-value & IDI & p-value & Hosmer and Lemeshow test & AIC & $\begin{array}{l}\text { Bootstrap- } \\
\text { corrected } \\
\text { C-statistics }\end{array}$ \\
\hline $\mathrm{ACEF}+\mathrm{CK}-\mathrm{MB}$ & 0.733 & 0.046 & 0.459 & $<0.001$ & 0.018 & 0.017 & $x^{2}=8.75, p=0.364$ & 440.24 & 0.731 \\
\hline ACEF score & 0.680 & & & & & & $x^{2}=3.39, p=0.907$ & 452.91 & 0.680 \\
\hline Mehran A+CK-MB & 0.727 & 0.091 & 0.459 & $<0.001$ & 0.017 & 0.021 & $x^{2}=7.02, p=0.534$ & 440.33 & 0.725 \\
\hline Mehran score A & 0.694 & & & & & & $x^{2}=5.09, p=0.748$ & 451.33 & 0.695 \\
\hline Mehran B + CK-MB & 0.734 & 0.102 & 0.459 & $<0.001$ & 0.018 & 0.020 & $x^{2}=9.31, p=0.317$ & 436.60 & 0.731 \\
\hline Mehran score B & 0.704 & & & & & & $x^{2}=7.07, p=0.529$ & 447.95 & 0.702 \\
\hline
\end{tabular}

CK-MB creatine kinase isoenzymes/creatine kinase-MB, ACEF Age, creatinine and ejection fraction, REICIN REduction of rlsk for Contrast-Induced Nephropathy

Table 4 Discrimination and calibration analysis of CK-MB added to models in PRECOMIN study data set

\begin{tabular}{|c|c|c|c|c|c|c|c|c|c|}
\hline & C-statistics & p-value & NRI & $p$-value & IDI & $p$-value & Hosmer and Lemeshow test & AIC & $\begin{array}{l}\text { Bootstrap- } \\
\text { corrected } \\
\text { C-statistics }\end{array}$ \\
\hline$A C E F+C K-M B$ & 0.724 & 0.059 & 0.236 & $<0.001$ & 0.015 & $<0.001$ & $x^{2}=8.93, p=0.348$ & 440.24 & 0.723 \\
\hline ACEF score & 0.700 & & & & & & $x^{2}=5.62, p=0.690$ & 340.16 & 0.699 \\
\hline Mehran A+CK-MB & 0.742 & 0.024 & 0.236 & $<0.001$ & 0.011 & 0.027 & $x^{2}=3.90, p=0.866$ & 329.31 & 0.737 \\
\hline Mehran score A & 0.716 & & & & & & $x^{2}=6.40, p=0.602$ & 334.14 & 0.714 \\
\hline Mehran B + CK-MB & 0.718 & 0.002 & 0.236 & $<0.001$ & 0.012 & 0.026 & $x^{2}=8.40, p=0.395$ & 336.98 & 0.714 \\
\hline Mehran score B & 0.680 & & & & & & $x^{2}=5.31, p=0.725$ & 342.46 & 0.680 \\
\hline
\end{tabular}

$C K-M B$ creatine kinase isoenzymes/creatine kinase-MB, ACEF Age, creatinine and ejection fraction, PRECOMIN PREdictive Value of COntrast voluMe to creatinine Clearance Ratio

with CI-AKI (odds ratio [OR]: 3.21, 95\% CI: 1.89-5.46, $p<0.001$, Table2). In multivariable logistic regression, log-transformed CK-MB $>4.7$ had an independent association with CI-AKI (adjusted OR: 3.40, 95\% CI: 1.935.98, $p<0.001$, Table 2). Other variables associated with CI-AKI included age, Scr and LVEF. Table 2 depicts the performance of each covariate in logistic regression analysis.

\section{Discrimination and calibration analysis of CK-MB added to models}

The addition of the categorical variable log-transformed $\mathrm{CK}-\mathrm{MB}$ to ACEF score, Mehran score A and Mehran score B did contribute to increase in C-statistics, which ranged from 0.680 to $0.733(p=0.046), 0.694$ to 0.727 $(p=0.091), 0.704$ to 0.734 ( $p=0.102)$, respectively (Fig. 1 , Table 3). And the CK-MB added significant discriminative value to the traditional models when evaluated by NRI and IDI (Table 3). These models embodied a good calibration for CI-AKI based on the Hosmer-Lemeshow test (Table 3). By internal bootstrap validation, the bootstrap-corrected C-statistics ranged from 0.680 to 0.731 , 0.695 to $0.725,0.702$ to 0.731 , respectively (Table 3 ). Additionally, the external validation data set had similar good performance in discrimination and calibration (Table 4, Fig. 2). The external validation shown the addition of CK-MB to ACEF score, Mehran score A and Mehran score $\mathrm{B}$ did contribute to increase in C-statistics, which ranged from 0.700 to $0.724(p=0.059), 0.716$ to $0.742(p=0.024), 0.680$ to $0.718(p=0.002)$, respectively (Table 4).

\section{Discussion}

In this multicenter, prospective study of MI patients who underwent CAG or PCI, we found that preprocedural peak CK-MB was an independent predictor of CI-AKI. The risk of CI-AKI was over 3.4-fold among patients with log-transformed $\mathrm{CK}-\mathrm{MB}>4.7$ than those without. The addition of the variable CK-MB to either ACEF score or MRS did result in increasing CI-AKI risk demonstrated by $\mathrm{C}$-statistics. And the $\mathrm{CK}-\mathrm{MB}$ added significant discriminative value to the traditional models when assessed by NRI and IDI. All the results suggested CK-MB plays a key role in the clinical predictive value for CI-AKI among MI patients undergoing CAG or PCI.

In concordance with our results, one previous study recognized increased CK-MB and RDW levels were 

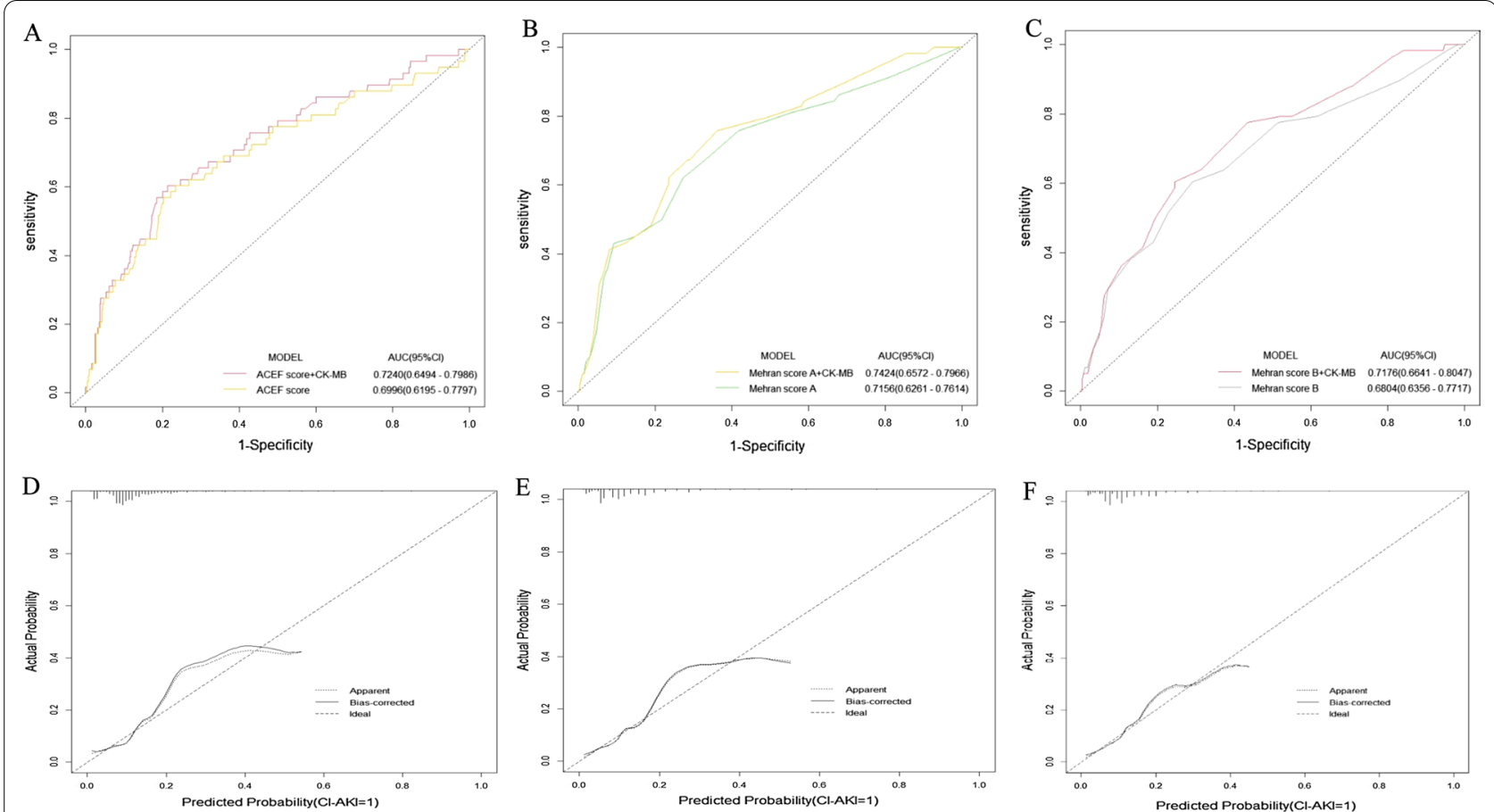

Fig. 2 The performance of CK-MB before and after adding ACEF score and MRS in PRECOMIN study data set. a The ROC curves of ACEF sCore and $\mathrm{ACEF}$ score + CK-MB for Cl-AKI. $\mathbf{b}$ The ROC curves of Mehran score A and Mehran score A+CK-MB for Cl-AKI. c The ROC curves of Mehran score B and Mehran score B + CK-MB for Cl-AKI. d The bootstrap curve of ACEF score + CK-MB integrated model for CI-AKI. e The bootstrap curve of Mehran score A + CK-MB integrated model for Cl-AKI. f The bootstrap curve of Mehran score B + CK-MB integrated model for Cl-AKI. AUC area under the receiver operating characteristic curve, $R O C$ receiver operator characteristic, $C K-M B$ creatine kinase isoenzymes/creatine kinase-MB, MRS Mehran risk score, ACEF Age, creatinine and ejection fraction, PRECOMIN PREdictive Value of COntrast voluMe to creatinine Clearance Ratio

significantly associated with CI-AKI among AMI patients. AMI patients with CK-MB $>55 \mathrm{U} / \mathrm{L}$ were $1.2-$ fold more likely to develop CI-AKI than those without [8]. However, that single-center retrospective study, with a relatively small amount of AMI patients, did not research the independent predictive value of CK-MB for CI-AKI and the correlation between CK-MB and long-term prognosis. Our multicenter prospective study, including 1131 nonselective MI population, described both the independent predictive value of CK-MB for $\mathrm{CI}-\mathrm{AKI}$ and the association of CK-MB with long-term prognosis.

Luis et.al. indicated that an independent association was not enough to establish the usefulness of a biomarker. They suggested inserting a new variable into a traditional risk scoring tool, and then comparing the performance of the traditional predictive model with that of an alternative model [14]. The ACEF score, a traditional risk model, has already been considered as a risk scoring of CI-AKI among patients undergoing primary PCI for a user-friendly clinical parameter by a quick preprocedural prediction of CI-AKI $[10,15,16]$. The MRS was another forecasting tool widely used to stratify the probability of developing CI-AKI after PCI [11]. After integrating variable CK-MB with the traditional risk models, we found the C-statistics significantly increased.

The mechanisms underlying the prediction of CI-AKI by $\mathrm{CK}-\mathrm{MB}$ may be related to hemodynamic instability. The elevation of preprocedural CK-MB, indicating the extent of myocardial necrosis, was closely related to the occurrence of cardiogenic shock and heart failure [17-19]. A consequent decrease in cardiac output led to a decline of renal perfusion as well as renal ischemia, resulting in AKI ultimately. Although troponin $\mathrm{T}$ and troponin I were more sensitive than CK-MB in detecting minor myocardial damage, measurement of CK-MB may be used to provide a facile clinical estimation of the infarct size [20]. Furthermore, troponin $\mathrm{T}$ and troponin I have not been uniformly used in low- and middleincome countries in the past clinical practice, which may result in bias due to different detection quality. Moreover, there are defects in Roche's high quality tests, which are more expensive than tests of CK-MB.

Our study also found that patients with log-transformed CK-MB > 4.7 had lower LVEF than those without. 
A decrease of LVEF indicated the loss of contractility due to acute ischemia or myocardial necrosis [21, 22]. Several studies have indicated that worsened LVEF was a predictor of CI-AKI [23-26]. In accordance with the previous study $[23,27,28]$, our study demonstrated that the age and basal creatinine were independently correlated with CI-AKI. Cinar T et al. also indicated that the age, creatinine and ejection fraction score correlated with ST-elevation myocardial infarction-related cardiogenic shock [29]. CK-MB might be a promising and timely tool for predicting CI-AKI among such MI patients. Therefore, regular monitoring, preventive strategies, and even priority treatment should be given to patients with logtransformed CK-MB > 4.7 for a well renal outcome in MI patients.

\section{Limitation}

First, the definition of CI-AKI was diverse. We adopted a definition of CI-AKI $\mathrm{I}_{0350}$ based on the increase in Scr, and used both baseline and postprocedural values, which only gave a moderately accurate evaluation of renal function. However, the definition of CI-AKI in our research was commonly cited in previous studies. Second, the logtransformed CK-MB may be complex in clinical applications. This defect will limit to generalize our results. Third, since there were 855 missing data of troponin $\mathrm{T}$ and 822 missing data of troponin I in our study, it is hard to detect the predictive value of troponin for CI-AKI. Fourth, the single center in PRECOMIN study is one of the centers in REICIN study, but the subjects in the two studies were enrolled at different periods.

\section{Conclusion}

The present study might be the first to report that the preprocedural peak CK-MB is a powerful indicator of CI-AKI among MI patients. The application of such a readily available biomarker may help clinicians to make a judgment on the CI-AKI risk of the MI patients. Logtransformed $\mathrm{CK}-\mathrm{MB}>4.7$ may be an optimal target for patients to receive therapeutic measures to prevent CI-AKI.

\footnotetext{
Abbreviations

CK-MB: Creatine kinase MB; Cl-AKI: Contrast-induced acute kidney injury; AKI: Acute kidney injury; MI: Myocardial infarction; AMI: Acute myocardial infarction; CAG: Coronary angiography; PCl: Percutaneous coronary intervention; RDW: Red Cell Distribution Width; ACEF: Age, creatinine and ejection fraction; MRS: Mehran risk score; LVEF: Left ventricular ejection fraction; CHF: Congestive heart failure; DM: Diabetes mellitus; IABP: Intra-aortic balloon pump; CKD Chronic kidney disease; GLU: Blood glucose; Hb: Hemoglobin; hct: Hematocrit; Scr: Serum creatinine; CCR: Creatinine Clearance Rate; eGFR: Estimated glomerular filtrationrate; BUN: Blood urea nitrogen; HDL-C: High density lipoprotein cholesterol; ALB: Albumin; ACEl: Angiotensin-converting enzyme inhibitor; ARB: Angiotensin receptor blocker; CCB: Calcium channel blocker; ROC: Receiver operating characteristic; NRI: Net reclassification index; IDI:
}

Integrated discrimination and improvement; REICIN: REduction of rlsk for Contrast-Induced Nephropathy; PRECOMIN: PREdictive Value of COntrast voluMe to creatinine Clearance Ratio.

\section{Acknowledgements \\ None.}

\section{Authors' contributions}

Substantial contributions to the conception and design of the study (SQC,KHC, YL, JL); data collection (YBH, BW, HZH, QL, MFL); data analysis and/ or interpretation of data for the work (LYZ, DYX, ZDH); drafting of the work or revising it critically for important intellectual content (WW, YHZ, RHT, MZ); final approval of the version to be published (all the authors). All authors read and approved the final manuscript.

\section{Funding}

This study was supported by grants from Beijing Lisheng Cardiovascular Health Foundation and Guangdong Provincial People's Hospital Foundation (LHJJ20141751), National Science Foundation of China (81970311), Guangdong Provincial Science and Technology Plan Project (2017B030314041) and Guangdong Provincial Fund for Clinical Medications (2019ZH01). The funders had no role in the study design, data collection and analysis, decision to publish, or preparation of the manuscript; the work was not funded by any industry sponsors.

\section{Availability of data and materials}

Data relevant to this study are available from the corresponding authors upon reasonable request.

\section{Declarations}

\section{Ethics approval and consent to participate}

The study was approved by the Research Ethics Committee of Guangdong Provincial People's Hospital, Guangdong Academy of Medical Sciences (No. GDREC2012141H). All the patients enrolled in the study signed written informed consent.

\section{Consent for publication}

Not applicable.

\section{Competing interests}

The authors have no conflicts of interest to disclose.

\section{Author details}

${ }^{1}$ Department of Cardiology, Guangdong Provincial Key Laboratory of Coronary Heart Disease Prevention, Guangdong Cardiovascular Institute, Guangdong Provincial People's Hospital, Guangdong Academy of Medical Sciences, Guangzhou 510080, China. ${ }^{2}$ Department of Endocrinology, Longyan First Affiliated Hospital of Fujian Medical University, Longyan 364000, China. ${ }^{3}$ The Second School of Clinical Medicine, Southern Medical University, Guangzhou 510515, China. ${ }^{4}$ Department of Cardiology, Maoming People's Hospital, Maoming 525000, China. ${ }^{5}$ Kunming Medical University, Kunming 650500, China. ${ }^{6}$ Department of Ultrasound Imaging, Yunnan Fuwai Cardiovascular Hospital, Kunming 650500, China. ${ }^{7}$ Guangdong Provincial People's Hospital, School of Medicine, South China University of Technology, Guangzhou 510100, China. ${ }^{8}$ Department of Cardiology, Longyan First Affiliated Hospital of Fujian Medical University, Longyan 364000, China.

Received: 25 March 2021 Accepted: 17 June 2021

Published online: 13 July 2021

\section{References}

1. Bae E, Kim JH, Jung MH, Jang SJ, Lee TW, Jung S, et al. Paricalcitol attenuates contrast-induced acute kidney injury by regulating mitophagy and senescence. Oxid Med Cell Longev. 2020. https://doi.org/10.1155/2020/ 7627934

2. Giacoppo D, Madhavan MV, Baber U, Warren J, Bansilal S, Witzenbichler B, et al. Impact of contrast-induced acute kidney injury after percutaneous 
coronary intervention on short- and long-term outcomes: pooled analysis from the HORIZONS-AMI and ACUITY trials. Circ Cardiovasc Interv. 2015;8(8): e002475. https://doi.org/10.1161/CIRCINTERVENTIONS.114. 002475 .

3. Nakahashi H, Kosuge M, Sakamaki K, Kiyokuni M, Ebina T, Hibi K, et al. Combined impact of chronic kidney disease and contrast-induced nephropathy on long-term outcomes in patients with ST-segment elevation acute myocardial infarction who undergo primary percutaneous coronary intervention. Heart Vessels. 2017;32(1):22-9. https://doi.org/10 1007/s00380-016-0836-8

4. Weisbord SD, Palevsky PM, Kaufman JS, Wu H, Androsenko M, Ferguson $\mathrm{RE}$, et al. Contrast-associated acute kidney injury and serious adverse outcomes following angiography. J Am Coll Cardiol. 2020;75(11):1311-20. https://doi.org/10.1016/j.jacc.2020.01.023.

5. Mehran R, Dangas GD, Weisbord SD. Contrast-associated acute kidney injury. N Engl J Med. 2019;380(22):2146-55. https://doi.org/10.1056/ NEJMra1805256.

6. Saito T, Matsumoto H, Matsuyama H, Sakai Y, Yamashita K, Kishi K, et al. Clinical evaluation of the new creatine kinase MB reagent kit "L-System CK-MB MtO." Rinsho Byori. 2011;59(3):236-42.

7. Bucholz EM, Whitlock RP, Zappitelli M, Devarajan P, Eikelboom J, Garg AX, et al. Cardiac biomarkers and acute kidney injury after cardiac surgery. Pediatrics. 2015;135(4):e945-956. https://doi.org/10.1542/peds.2014-2949.

8. Zbierska-Rubinkiewicz K, Trębacz O, Tomala M, Rubinkiewicz M, Chrzan I, Gackowski A. Creatine kinase-MB and red cell distribution width as predictors of contrast-induced nephropathy after percutaneous coronary intervention in acute myocardial infarction. Folia Med Cracov. 2017;57(3):87-99.

9. Moriyama N, Ishihara M, Noguchi T, Nakanishi M, Arakawa T, Asaumi $Y$, et al. Admission hyperglycemia is an independent predictor of acute kidney injury in patients with acute myocardial infarction. Circ J. 2014;78(6):1475-80. https://doi.org/10.1253/circj.cj-14-0117.

10. Andò G, Morabito G, de Gregorio C, Trio O, Saporito F, Oreto G. The ACEF score as predictor of acute kidney injury in patients undergoing primary percutaneous coronary intervention. Int J Cardiol. 2013;168(4):4386-7. https://doi.org/10.1016/j.ijcard.2013.05.049.

11. Mehran R, Aymong ED, Nikolsky E, Lasic Z, lakovou I, Fahy M, et al. A simple risk score for prediction of contrast-induced nephropathy after percutaneous coronary intervention: development and initial validation J Am Coll Cardiol. 2004;44(7):1393-9. https://doi.org/10.1016/j.jacc.2004. 06.068 .

12. Liu Y, Chen JY, Tan N, Zhou YL, Yu DQ, Chen ZJ, et al. Safe limits of contrast vary with hydration volume for prevention of contrast-induced nephropathy after coronary angiography among patients with a relatively low risk of contrast-induced nephropathy. Circ Cardiovasc Interv. 2015. https:// doi.org/10.1161/CIRCINTERVENTIONS.114.001859.

13. Riley RD, Ensor J, Snell K, Harrell FE Jr, Martin GP, Reitsma JB, et al. Calculating the sample size required for developing a clinical prediction model. BMJ. 2020. https://doi.org/10.1136/bmj.m441.

14. Correia LC, Vasconcelos I, Garcia G, Kalil F, Ferreira F, Silva A, et al. Does C-reactive protein add prognostic value to GRACE score in acute coronary syndromes. Arq Bras Cardiol. 2014;102(5):449-55. https://doi.org/10. 5935/abc.20140056.

15. Liu YH, Liu Y, Zhou YL, He PC, Yu DQ, Li LW, et al. Comparison of different risk scores for predicting contrast induced nephropathy and outcomes after primary percutaneous coronary intervention in patients with ST elevation myocardial infarction. Am J Cardiol. 2016;117(12):1896-903. https://doi.org/10.1016/j.amjcard.2016.03.033.

16. Capodanno D, Ministeri M, Dipasqua F, Dalessandro V, Cumbo S, Gargiulo $\mathrm{G}$, et al. Risk prediction of contrast-induced nephropathy by ACEF score in patients undergoing coronary catheterization. J Cardiovasc Med (Hagerstown). 2016;17(7):524-9. https://doi.org/10.2459/JCM.0000000000 000215 .
17. Thiele H, Ohman EM, de Waha-Thiele S, Zeymer U, Desch S. Management of cardiogenic shock complicating myocardial infarction: an update 2019. Eur Heart J. 2019;40(32):2671-83. https://doi.org/10.1093/eurheartj/ ehz363.

18. Mebazaa A, Tolppanen H, Mueller C, Lassus J, DiSomma S, Baksyte G, et al. Acute heart failure and cardiogenic shock: a multidisciplinary practical guidance. Intensive Care Med. 2016;42(2):147-63. https://doi.org/10. 1007/s00134-015-4041-5.

19. Bahit MC, Kochar A, Granger CB. Post-myocardial infarction heart failure. JACC Heart Fail. 2018;6(3):179-86. https://doi.org/10.1016/j.jchf.2017.09. 015.

20. Hoshino T, Sakai Y, Yamashita K, Kishi K, Tanjoh K, Hirayama A, et al. Clinical evaluation of a new creatine kinase $M B$ activity reagent abrogating the effect of mitochondrial creatine kinase. Clin Lab. 2013;59(3-4):307-16. https://doi.org/10.7754/clin.lab.2012.120516.

21. Mello BH, Oliveira GB, Ramos RF, Lopes BB, Barros CB, Carvalho Ede O, et al. Validation of the Killip-Kimball classification and late mortality after acute myocardial infarction. Arq Bras Cardiol. 2014;103(2):107-17. https:// doi.org/10.5935/abc.20140091.

22. Ibanez B, James S, Agewall S, Antunes MJ, Bucciarelli-Ducci C, Bueno H, et al. 2017 ESC Guidelines for the management of acute myocardial infarction in patients presenting with ST-segment elevation: the Task Force for the management of acute myocardial infarction in patients presenting with ST-segment elevation of the European Society of Cardiology (ESC). Eur Heart J. 2018;39(2):119-77. https://doi.org/10.1093/eurheartj/ ehx393.

23. Yao ZF, Shen H, Tang MN, Yan Y, Ge JB. A novel risk assessment model of contrast-induced nephropathy after percutaneous coronary intervention in patients with diabetes. Basic Clin Pharmacol Toxicol. 2021;128(2):30514. https://doi.org/10.1111/bcpt.13501.

24. Khoury S, Steinvil A, Gal-Oz A, Margolis G, Hochstatd A, Topilsky Y, et al. Association between central venous pressure as assessed by echocardiography, left ventricular function and acute cardio-renal syndrome in patients with ST segment elevation myocardial infarction. Clin Res Cardiol. 2018;107(10):937-44. https://doi.org/10.1007/s00392-018-1266-7.

25. Chyrchel M, Hałubiec P, Łazarczyk A, Duchnevič O, Okarski M, Gębska M, et al. Low ejection fraction predisposes to contrast-induced nephropathy after the second step of staged coronary revascularization for acute myocardial infarction: a retrospective observational study. J Clin Med. 2020. https://doi.org/10.3390/jcm9061812.

26. Santos PR, Carneiro Neto JD, Arcanjo FP, Carneiro JK, Carneiro RC, do Amaral CL. Contrast-induced nephropathy after primary angioplasty for acute myocardial infarction. J Bras Nefrol. 2015;37(4):439-45. https://doi. org/10.5935/0101-2800.20150070.

27. Çınar T, Karabağ Y, Ozan Tanık V, Çağdaş M, Rencüzoğulları I, Öz A. The investigation of TIMI risk index for prediction of contrast-induced acute kidney injury in patients with ST elevation myocardial infarction. Acta Cardiol. 2020;75(1):77-84. https://doi.org/10.1080/00015385.2018.15512 63.

28. Efe SC, Keskin M, Toprak E, Arslan K, Öz A, Güven S, et al. A novel risk assessment model using urinary system contrast blush grading to predict contrast-induced acute kidney injury in low-risk profile patients. Angiology. 2021. https://doi.org/10.1177/00033197211005206.

29. Çinar T, Hayiroğlu Mi, Şeker M, Doğan S, Çiçek V, Öz A, et al. The predictive value of age, creatinine, ejection fraction score for in-hospital mortality in patients with cardiogenic shock. Coron Artery Dis. 2019;30(8):569-74. https://doi.org/10.1097/MCA.0000000000000776.

\section{Publisher's Note}

Springer Nature remains neutral with regard to jurisdictional claims in published maps and institutional affiliations. 